\title{
Modeling the Cut-off Frequency of Acoustic Signal with an Adaptative Neuro-Fuzzy Inference System (ANFIS)
}

\author{
Y. Nahraoui , E.H. Aassif, \\ LMTI, Faculté des sciences,Université Ibn \\ Zohr, Agadir, Maroc
}

\author{
G.Maze \\ LOMC, Université du Havre, \\ Institut Universitaire de Technologie, \\ Place Robert Schuman, 76610 Le Havre, \\ France
}

\author{
R.LATIF \\ ESSI, Ecole Nationale des Sciences \\ appliquées, Université Ibn Zohr, \\ Agadir, Maroc
}

\begin{abstract}
An Adaptative Neuro-Fuzzy Inference System (ANFIS), new flexible tool, is applied to predict the cut-off frequencies of the symmetric and the anti-symmetric circumferential waves $\left(S_{i}\right.$ and $\left.A_{i}, i=1,2\right)$ propagating around an elastic aluminum cylindrical shell of various radius ratio $b / a(a$ : outer radius and $b$ : inner radius). The time-frequency of WignerVille and the proper modes theory are used in this study to compare and valid the frequencies values predicted by the ANFIS model. The useful data, of the cut-off frequencies $(\mathrm{ka})_{c}$, are used to train and to test the performances of the model. These data are determined from the values calculated using the proper modes theory of resonances and also from those determined using the time-frequency images of Wigner-Ville. The material density, the radius ratio $b / a$, the index $i$ of the symmetric and the antisymmetric circumferential waves, and the longitudinal and transverse velocities of the material constituting the tube, are selected as the input parameters of the ANFIS model. This technique is able to model and to predict the cut-off frequencies, of the symmetric and the anti-symmetric circumferential waves, with a high precision, based on different estimation errors such as mean relative error (MRE), mean absolute error (MAE) and standard error (SE). A good agreement is obtained between the output values predicted using the propose model and those computed by the proper modes theory.
\end{abstract}

Keywords-ANFIS; time-frequency; SPWV; Acoustic scattering, acoustic circumferential waves; cut-off frequency;cylindrical shell.

\section{INTRODUCTION}

In a previous studies [1, 2], we have analysed the acoustic signal scattered by a thin elastic tube immersed in water using the time-frequency representation of Wigner-Ville. The Wigner-Ville image obtained in these analyses allowed to determine the cut-off frequency, of the anti-symmetric circumferential waves A1 propagating around the aluminum cylindrical shell of different radius ratio $b / a$. These analyses permitted also to determine, form the time-frequency image, the thickness of elastic cylindrical shell.

Many studies, theoretical and experimental, showed that acoustic resonances of a cylindrical shell are related to its physical and geometrical properties. Conversely, starting from the resonances of circumferential waves we can characterize material constituting a cylindrical shell the geometry of which is known [1-6].

The resonances of the symmetric and the anti-symmetric circumferential waves ( $S_{i}$ and $A_{i}, i=0,1,2, \ldots$ index of the mode) are observed on the spectrum of the acoustic pressure backscattered by the cylindrical shell [7]. Apart from the specular reflection, the backscattered pressure field results mainly from the interactions of different kinds of creeping waves that generate resonances in the spectrum. The resonance frequencies of the circumferential waves $\left(S_{i}\right.$ and $\left.A_{i}\right)$ essentially depend on the radius ratio $b / a$. Using the proper modes theory, we can determine the cut-off frequencies of the symmetric and the anti-symmetric circumferential waves $\left(S_{i}\right.$ and $A_{i}, i=1,2$ ) for a aluminium cylindrical shell with different radius ratio b/a. One of the most important points is find out some parameters that carry most of the information available from the response of the cylindrical shell. Such parameters may be found from the velocity dispersion of the circumferential waves $\left(S_{i}\right.$ and $\left.A_{i}\right)$, since it is directly related to the geometry and to the physical properties of the shell.

Different methods have been proposing for analyse of the circumferential waves propagating around the cylindrical shell which includes temporal analysis [7,9], spectrum analysis [5, 7], parametric time-frequency analysis [10-16], wavelet transform [19-20] and neural networks [21-22].

The present paper is especially concerned with the soft computing technique such as fuzzy logic system. The adaptative neuro-fuzzy inference system (ANFIS) is selected and applied to predict the cut-off frequencies of the symmetric and the anti-symmetric circumferential waves $\left(S_{i}\right.$ and $A_{i}$, $i=1,2, \ldots$ ) for cylindrical shell of various radius ratio $b / a$ that cannot be measured experimentally. The cut-off frequencies obtained from the computed values using the proper modes are used as data in the ANFIS model. In experiments, the timefrequency representation of Wigner-Ville of the acoustic signal backscattered by cylindrical shell is calculated. The ANFIS model and the Wigner-Ville technique are tools for the statistical analysis, making possible the construction of a model of behavior starting from a certain number of examples. The model is able to predict the cut-off frequencies of the symmetric and the anti-symmetric circumferential waves $\left(\mathrm{S}_{\mathrm{i}}\right.$ and $\left.\mathrm{A}_{\mathrm{i}}, \mathrm{i}=1,2 \ldots\right)$ for aluminum cylindrical shell of various 
radius ratio $b / a$. The radius ratios used, in this paper, are between 0.4 and 0.99 . The cut-off frequencies values determined using the ANFIS model are compared with those determined from the time-frequency images of Wigner-Ville to validate the robustness of the model proposed. In this study, we have use three aluminum cylindrical shell of various radius radio b/a $(0.9,0.95$ and 0.97$)$. These examples are used to evaluate the performance and robustness of the ANFIS model and make a comparison with the analysis of time-frequency Wigner-Ville to determine the dimensional radius ratio of the cylindrical shell studied.

\section{BACKSCATTERING RESPONSE FROM A CYLINDRICAL SHELL}

\section{A. Acoustic scatring by an air-filled cylindrical shell}

The analysis of acoustic signals scattered by an air-filled cylindrical shell immersed in water is a topic that has received large attention for several years [1-8]. In previous studies, the characterization of the scattering problem is mainly performed in the frequency domain. The module of the backscattered pressure in the faraway field, called "form function", by the cylindrical shell can be derived directly from a computational model $[7,11]$.

This module is also called a backscattered spectrum. Apart from the specular reflection, the backscattered pressure field results mainly from the interactions of different kinds of creeping waves that generate "resonances" in the spectrum. These resonances are in relation with the symmetric and antisymmetric circumferential waves (S0, A1, S1, S2, A2,..).

The scattering of an infinite plane wave by an air-filled cylindrical shell of radii ratio $b / a$ is investigated through the solution of the wave equation and the associated boundary conditions.

Fig. 1 shows the cylindrical coordinate orientation and the direction of a plane wave incident on an infinitely long cylindrical shell in a fluid medium. The fluid (1) inside the shell has a density of $\rho_{1}$ and propagation velocity $c$. In general, the outer fluid (2) will be different and is described by the parameters $\rho$ and $c$. The parameters for the two fluids outside and inside the shell are given in Table 1.

The axis of the cylindrical shell is taken to be the $z$-axis of the cylindrical coordinate system $(r, \theta, z)$. Let a plane wave incident on an infinite cylindrical shell with air-filled cavity (fluid 2), be submerged in water (fluid 1), see figure 1.

The backscattered complex pressure $P_{\text {diff }}$ by a cylindrical shell in a faraway field $(r \gg \rightarrow$, we have neglected the diffraction of waves and one receives only the part backscattered of the complex pressure field) is the summation of the incident wave, the reflective wave (1), surface waves tell shell waves (2) (whispering Gallery, Rayleigh, ...) and Scholte waves (A) (3) connected to the geometry of the object (figure 2). The waves (2) and (3) are the circumferential waves. For these waves one distinguishes the waves $\mathrm{A}$, the symmetric waves S0, S1, S2 and the anti-symmetric waves A1, A2.

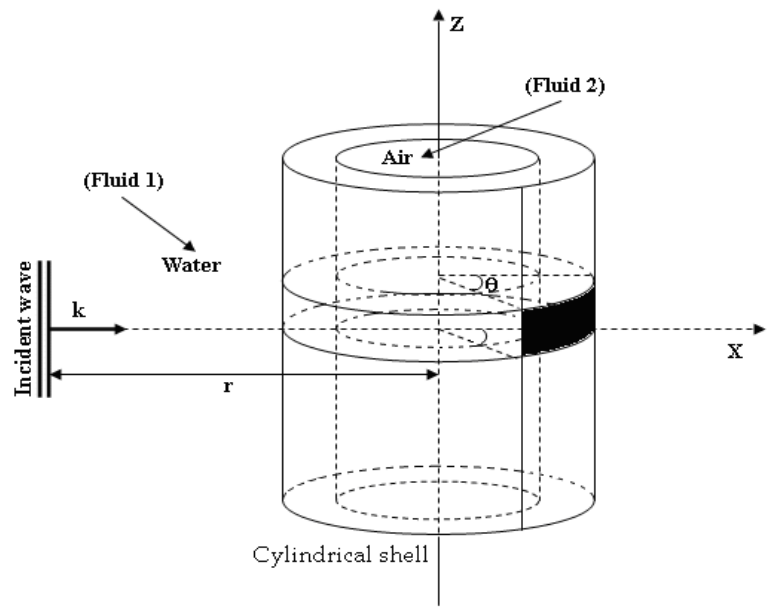

Fig.1. Geometry used for formulating the sound backscattering from a cylindrical shell

The general form of the backscattered pressure field at normal incidence can be expressed as [7-8, 23]

$$
P_{\text {diff }}(\omega)=P_{0} \sum_{n=0}^{\infty} \frac{D_{n}^{(1)}(\omega)}{D_{n}(\omega)} H_{n}^{(1)}(k r)
$$

Where $\omega=2 \pi f$ is the angular frequency, $k$ the wave number with respect to the wave velocity in the external fluid and $P_{0}$ the amplitude of the plane incident wave. $\mathrm{D}_{\mathrm{n}}^{1}(\omega)$ and $\mathrm{D}_{\mathrm{n}}(\omega)$ are determinants computed from the boundary conditions of the problem (continuity of stress and displacement at both interfaces). The function $\mathrm{H}_{\mathrm{n}}^{(1)}$ is the Hankel function of the first kind.

The module of the backscattered complex pressure in a faraway field is called form function. This function is obtained by the relation $[7-8,23]$

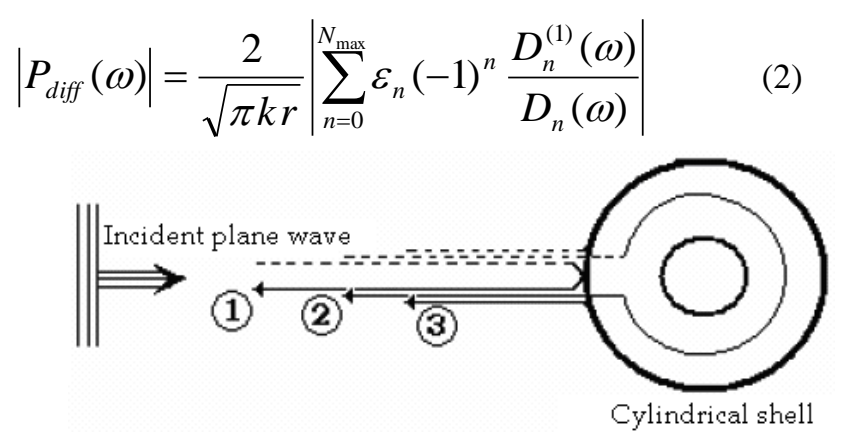

Fig.2. Mechanisms of the formation of echoes showing the specular reflection (1) and shell waves (2) and Scholte wave (A) (3).

where is the Neumann factor $\left(\varepsilon_{n}=1\right.$, if $n=0 ; \varepsilon_{n}=2$, if $n>$ $0), k=\omega / c$ is the incident wave number and $c$ is the phase velocity in water.

The physical parameters used in the calculation of the backscattered complex pressure are illustrated in table I. 
TABLE I. PHYSICAL PARAMETERS

\begin{tabular}{lllc}
\hline \hline & $\begin{array}{l}\text { Density } \\
\rho\left(\mathrm{kg} / \mathrm{m}^{3}\right)\end{array}$ & $\begin{array}{l}\text { Longitudinal } \\
\text { Velocity } c_{L}(\mathrm{~m} / \mathrm{s})\end{array}$ & $\begin{array}{c}\text { Transverse } \\
\text { Velocity } c_{T}(\mathrm{~m} / \mathrm{s})\end{array}$ \\
\cline { 2 - 4 } Aluminum & 2790 & 6380 & 3100 \\
Water & 1000 & 1470 & - \\
Air & 1.29 & 334 & - \\
\hline \hline
\end{tabular}

The figure 3 shows the module of the backscattered complex pressure in function of the reduced frequency $k a$ (without unit) given by :

$$
k a=\frac{\omega a}{c}=\frac{2 \pi}{c\left(1-\frac{b}{a}\right)} f d
$$

Where $d=a-b$ is the thickness of a cylindrical shell and $f$ is the frequency of resonance of a wave in $\mathrm{Hz}$.

The temporal signal response $P(t)$ of a cylindrical shell is computed by taking the Inverse of Fourier Transform of the module of the backscattered complex pressure:

$$
P(t)=\frac{1}{2 \pi} \int_{-\infty}^{+\infty} h(\omega) P_{d i f f}(\omega) e^{-i \omega t} d \omega
$$

Where $h(\omega)$ is a smoothing window.

The succession of shell resonances (corresponding to frequency of resonances) in the spectrum of the figure 3 is connected with the propagation of acoustic circumferential waves: Scholte wave (A) and shell waves (S0, A1, S1, S2, A2, ...). The temporal signal backscattered by an Aluminum cylindrical shell is obtained by the Inverse Transform Fourier

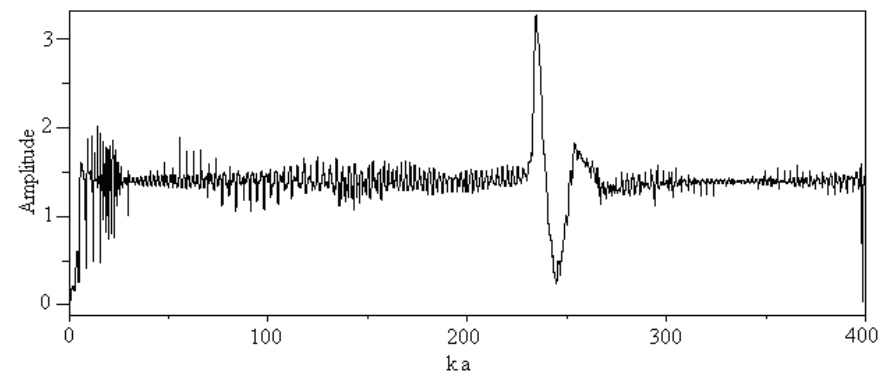

Fig.3. Module of the backscattered complex pressure for an infinite aluminum cylindrical shell with air-filled cavity of radii radio $b / a=0.95$

of the module of the backscattered complex pressure using the equation (4). The figure 4 presents this signal and shows the specular reflection (1) (large amplitude and short duration) and several wave packets (2) and (3) associated with different circumferential waves (A, S0, A1, S1, S2, A2, ...). The observation of this signal shows a succession of components more or less distinct that one seeks then to identify. The different echoes finish by overlapping and in these conditions, the identifications and measures of arrival times of echoes (this time depends on the radii of the tube $a$ and $b$ ) become difficult, perhaps impossible. This constitutes a major disadvantage of the temporal approach. An important feature of the acoustic circumferential waves is the velocity dispersion that leads to a time spreading of wave packets.

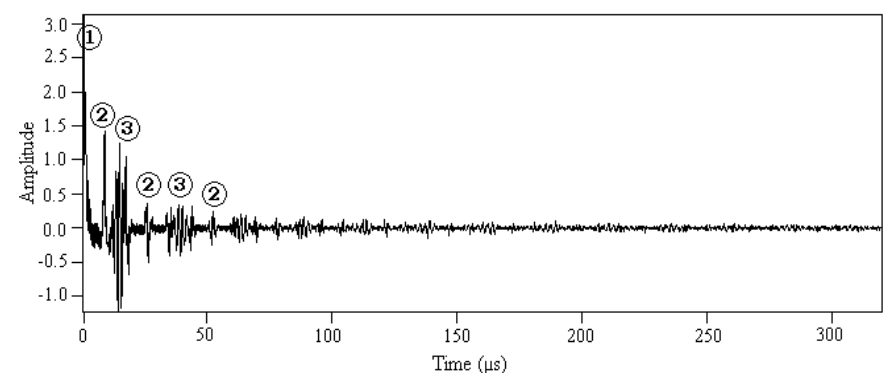

Fig.4. Signal backscattered by an aluminum cylindrical shell with airfilled cavity, $b / a=0.95$ (Specular reflection echo (1), shell waves echoes (2) and Scholte wave echo (A) (3)).

\section{B. Dispersion and cut-off frequency determined using the proper modes theory}

An important feature of the circumferential waves is the velocity of dispersion that leads to a time spreading of wave packets (shell of waves). In the case of the circumferential waves (2) for instance, the dispersion velocity is significant and the time spreading is much more important than in the case of (3). Resonances that appear on the backscattered pressure field are linked to the propagation of circumferential waves around the tube. One finds the wave of Scholte $(A)$ and the waves of shell (SO, A1, S1, S2, A2).

The group velocity of circumferential waves is estimated from the resonance frequencies, using the proper modes theory, that correspond to the circumferential waves. The calculation of the resonance frequencies of these waves have been made by the cancellation of the determinant $D$ given by [8]:

$$
\mathrm{D}=\left[\begin{array}{llll}
d_{11} & d_{12} & d_{13} & d_{14} \\
d_{21} & d_{22} & d_{23} & d_{24} \\
d_{31} & d_{32} & d_{33} & d_{34} \\
d_{41} & d_{42} & d_{43} & d_{44}
\end{array}\right]
$$

where the 16 non-vanishing elements $d_{\mathrm{ij}}$ can all be determined from the boundary conditions of the problem, and they have all been listed elsewhere [2]. The resolution of the equation $D=0$ allows to determine the different proper modes for each type of the symmetric and the anti-symmetric circumferential waves $\left(S_{i}\right.$ and $\left.A_{i}, i=0,1,2, \ldots\right)$. Once frequencies of resonances are determined, we calculate the difference $\Delta$ $k a$ between two successive resonance frequencies. The group velocity of the symmetric and the anti-symmetric circumferential waves for each frequency is given by [8]:

$$
c_{g}=c \Delta k a
$$

where $\Delta k a$ the gap between two successive resonances.

The figure 5 shows the evolution of the group velocity in function of the $(\mathrm{ka})$ for different waves. 
Starting from the similitude that exists between the circumferential waves in the case of a thin elastic tube and the Lamb waves in the case of a plaque of the same thickness, it is possible to use the classical relations on the Lamb waves to ascend to the value of the reduced cut-off frequency of circumferential waves in the case of a tube $[2-5,14-15,24-$ 25].

In the case of a thin plaque, the cut-off frequencies of the symmetric and anti-symmetric Lamb waves are given by $[2$, 14]:

$$
(f d)_{c}=\left\{\begin{array}{l}
m_{s} c_{T} \\
\left(m_{s}+\frac{1}{2}\right) c_{L}
\end{array}\right.
$$

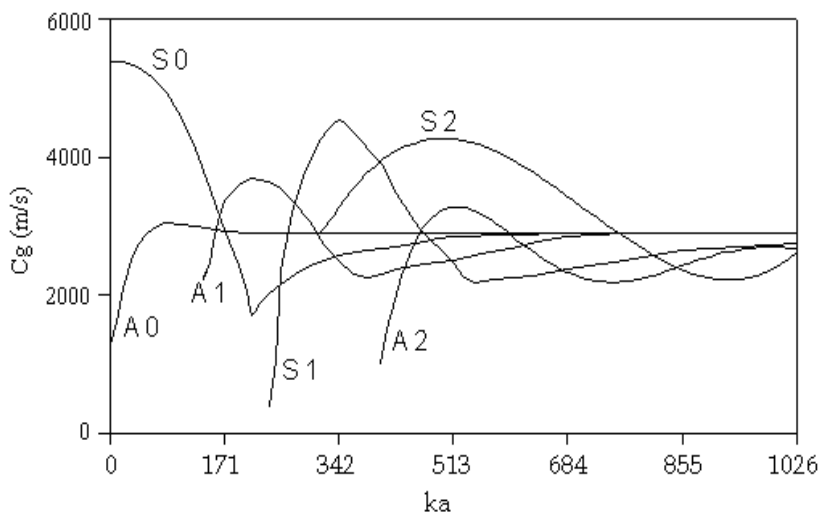

Fig.5. Dispersion velocity of the different circumferential waves of an aluminum cylindrical shell of radii radio $b / a=0.95$

$$
(f d)_{c}=\left\{\begin{array}{l}
m_{a} c_{L} \\
\left(m_{a}+\frac{1}{2}\right) c_{T}
\end{array}\right.
$$

where $c_{T}$ and $c_{L}$ are transverse and longitudinal velocities of the material constituting the cylindrical shell. The indices $s$ and $a$ on the integers $m$ number indicating symmetric and antisymmetric modes of plate vibrations respectively.

The cut-off frequencies, of the symmetric and antisymmetric circumferential waves, are determined by exploiting the equations 3,7 and $8[2,14]$ :

$$
\begin{aligned}
& (k a)_{c}=\frac{2 \pi}{c\left(1-\frac{b}{a}\right)}\left\{\begin{array}{l}
m_{s} c_{T} \\
\left(m_{s}+\frac{1}{2}\right) c_{L}
\end{array}\right. \\
& (k a)_{c}=\frac{2 \pi}{c\left(1-\frac{b}{a}\right)}\left\{\begin{array}{l}
m_{a} c_{L} \\
\left(m_{a}+\frac{1}{2}\right) c_{T}
\end{array}\right.
\end{aligned}
$$

where $m_{s}$ and $m_{a}$ (integers numbers) are the symmetric and anti-symmetric modes of circumferential waves respectively.
For the symmetric modes $S 1$ and $S 2$ the cut-off frequencies values are calculated from the equations (11) and (12) respectively:

For S1 mode:

$$
(k a)_{c}^{S 1}=\frac{2 \pi}{c\left(1-\frac{b}{a}\right)} \cdot c_{T}
$$

For $S 2$ mode:

$$
(k a)_{c}^{S 2}=\frac{\pi}{c\left(1-\frac{b}{a}\right)} \cdot c_{L}
$$

For the anti-symmetric modes $A 1$ and $A 2$ the cut-off frequencies values are calculated from the equations (13) and (14) respectively:

For Al mode:

$$
(k a)_{c}^{A 1}=\frac{\pi}{c\left(1-\frac{b}{a}\right)} \cdot c_{T}
$$

For $A 2$ mode:

$$
(k a)_{c}^{A 2}=\frac{3 \pi}{c\left(1-\frac{b}{a}\right)} \cdot c_{T}
$$

The calculated values, using the equations (11) to (14), of the cut-off frequencies of the symmetric and anti-symmetric circumferential waves $A 1, S 1, S 2$ and $A 2$ are given in table II.

TABLE II.

CUT-OFF FREQUENCIES VALUES OF DIFFERENT CIRCUMFERENTIAL WAVES FOR ALUMINUM CYLINDRICAL SHELL OF VARIOUS RADIUS RATIOS

\begin{tabular}{c||c|c|c|c|}
\cline { 2 - 5 } \multicolumn{1}{c||}{} & \multicolumn{4}{c|}{ Cut-off frequencies $(\mathrm{ka})_{c}$} \\
\cline { 2 - 5 } $\begin{array}{c}\text { Cylindrical } \\
\text { shell }\end{array}$ & $\begin{array}{c}\text { Mode A1 } \\
m_{a}=0\end{array}$ & $\begin{array}{c}\text { Mode S1 } \\
m_{s}=1\end{array}$ & $\begin{array}{c}\text { Mode S2 } \\
m_{s}=0\end{array}$ & $\begin{array}{c}\text { Mode A2 } \\
m_{a}=1\end{array}$ \\
\hline$b / a=0.9$ & 66.21 & 132.43 & 136.28 & 198.65 \\
\hline$b / a=0.95$ & 132.43 & 264.87 & 272.56 & 397.30 \\
\hline$b / a=0.97$ & 220.72 & 441.45 & 454.26 & 662.17 \\
\hline
\end{tabular}

\section{DISPERSION ANALYSIS USING TIME-FREQUENCY IMAGES}

The analysis of the returned echoes has traditionally been done in the frequency domain, and later in the time domain. A recent processing technique that seems to be gaining acceptance is to work in the combined time-frequency domain. Usually, projections of these three-dimensional surfaces are shown in the two-dimensional time-frequency plane. This evolution can be extracted from the echoes and displayed in as much detail as is feasible. Among the large number of existing time-frequency representations, some authors $[1-2,8,10-16]$ have proposed to use the Smoothed Pseudo Wigner-Ville. The 
choice of this particular distribution results from its interesting properties in terms of acoustic applications [1-2, 8, 12, 14-15].

\section{A. Theoretical fundamentals}

The Wigner-Ville distribution (WVD) of the real signal $x(t)$ is defined by $[4,8,11-14]$ :

$$
W V_{x}(t, v)=\int_{-\infty}^{+\infty} x\left(t+\frac{\tau}{2}\right) x^{*}\left(t-\frac{\tau}{2}\right) e^{-i 2 \pi v \tau} d \tau
$$

Time-frequency smoothing can then be applied to reduce the amplitude of these spurious terms. It can be achieved by using the Smoothed Pseudo Wigner-Ville (SPWV) [1-2]:

$$
\begin{aligned}
S P W V_{x}(t, f)= & \int_{-\infty}^{+\infty}\left|h\left(\frac{\tau}{2}\right)\right|^{2} \int_{-\infty}^{+\infty} g(t-u) x\left(u+\frac{\tau}{2}\right) \\
& \times x^{*}\left(u-\frac{\tau}{2}\right) \exp (-2 j \pi f \tau) d u d \tau
\end{aligned}
$$

The smoothing windows $g(t)$ and $h(t)$ are introduced into the $S P W V$ definition in order to allow a separate control of interferences either in time $(g)$ or in frequency $(h)$.

\section{B. Dispersion and cut-off frequency using $S P W V$}

Scattering from a finite object provides many interesting subjects for analysing the circumferential waves. For example, one of the challenging problems is how to determine the shape and physical properties of an object thanks to the SPWV. This technique appears to be a very useful tool for such a task, as it is able to represent a given signal simultaneously in time and frequency domains.

The resonances brought into evidence on the scattered complex pressure (figure 3 ) are linked to the propagation of circumferential waves: Scholte waves $(A)$ and shell waves ( $S O$, $A 1, S 1, S 2, A 2)$ in the case of a cylindrical shell with light thickness. In this study, one is interested only in the symmetric and the anti-symmetric circumferential waves $\left(S_{i}\right.$ and $A_{i}$, $i=1,2, \ldots)$. According to this spectrum (figure 3 ), the reduced frequencies scale in which appears the symmetric and the antisymmetric waves $\left(S_{i}\right.$ and $\left.A_{i}, i=1,2\right)$ are illustrated in the table III.

\section{TABLE III. RANGE FREQUENCIES OF CIRCUMFERENTIAL WAVES} FOR ALUMINUM CYLINDRICAL SHELL OF RADII RADIO $B / A=0.95$

\begin{tabular}{|c|c|}
\cline { 2 - 2 } \multicolumn{1}{c|}{} & Range frequencies $\left(k_{l} a\right)$ \\
\hline Anti-symmetric wave $A 1$ & $130-200$ \\
\hline Symmetric wave $S 1$ & $260-340$ \\
\hline Symmetric wave $S 2$ & $270-350$ \\
\hline Anti-symmetric wave $A 2$ & $>390$ \\
\hline
\end{tabular}

Figures 6, 7 and 8 represent the time-frequency images for the anti-symmetric circumferential wave $A l$ for aluminium cylindrical shell of various radius ratio $b / a$. When the time augments, the trajectory associated to anti-symmetric wave $A 1$, for each case (figures 6, 7 and 8), tends to an asymptotic value which equal the cut-off frequency $(k a)_{c}$ of this wave.
Using the proper modes theory, this frequency is calculated by the equation (13). More precisely, this cut-off frequency is the intersection point of the asymptotic trajectory of the antisymmetric wave $A l$ and the axis of frequencies (figures 6, 7 and 8). The values of the cut-off frequency $(k a)_{c}$ obtained from these images are presented in table IV. This table presents also those values computed with the proper modes theory (equation 13). We notice that the cut-off frequencies determined from the time-frequency images are in good concordance with those computed from proper modes theory $(P M T)$.

TABLE IV. COMPARISON BETWEEN THE CUT-OFF FREQUENCIES VALUES COMPUTED THEATRICALLY AND DETERMINED FROM $S P W V$ IMAGES FOR ANTI-SYMMETRIC CIRCUMFERENTIAL WAVE $A l$

\begin{tabular}{|l|c|c|}
\cline { 2 - 3 } \multicolumn{1}{c|}{} & \multicolumn{2}{c|}{ Cut-off frequencies $(\mathrm{ka})_{\mathrm{c}}$} \\
\hline Cylindrical shell & $\begin{array}{c}\text { Computed } \\
\text { using } P M T\end{array}$ & $\begin{array}{c}\text { Determined } \\
\text { using } S P W V\end{array}$ \\
\hline$b / a=0.9$ (figure 8) & 66.21 & $66.0 \pm 0.3$ \\
\hline$b / a=0.95$ (figure 7) & 132.43 & $132.0 \pm 0.3$ \\
\hline$b / a=0.97$ (figure 6) & 220.72 & $221.0 \pm 0.2$ \\
\hline
\end{tabular}

\section{MATERIALS AND METHOD}

\section{A. Fuzzy Inference System}

Fuzzy logic is an extension of Boolean logic that allows intermediate values between "True" and "False". In this approach the classical theory of binary membership in a set, is modified to incorporate the memberships between " 0 " and " 1 ". The fuzzy models are means of capturing humans expert knowledge about the process, in terms of fuzzy (if-then) rules.

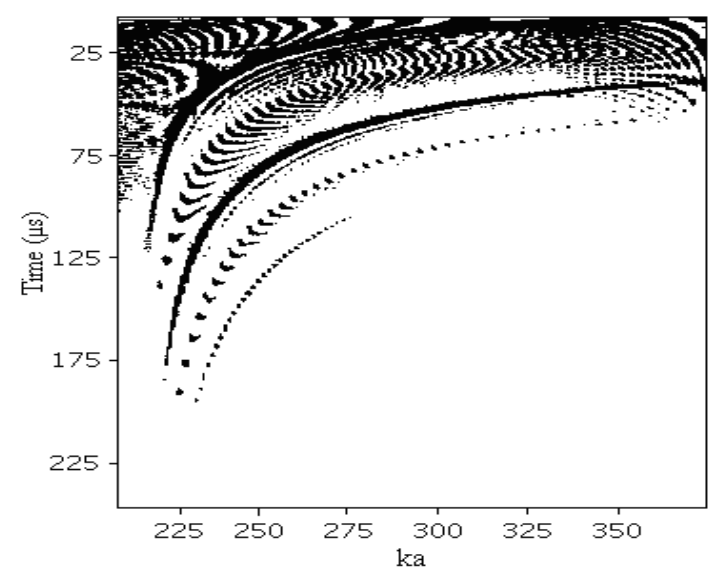

Fig.6. $\quad S P W V$ of backscattered signal for the first aluminum cylindrical shell of radii radio $b / a=0.97$ (Anti-symmetric circumferential wave $A 1$, $220<k a<375)$

The fuzzy inference system (FIS) can initialize and learn linguistic and semi-linguistic rules; hence it can be considered as direct transfer knowledge, which is the main advantage of fuzzy inference systems over classical learning systems and Neural Networks [26-28]. Often the rules of the fuzzy system are designated a priori and the parameters of the membership functions are adapted in the learning process from inputoutput data sets.

Basically, a fuzzy inference system is composed of five functional blocks, shown in Figure 9, as follows [26-28]: 
1) A rule base containing a number of fuzzy if-then rules. All the uncertainties, non linear relationships, or model complications are included in the descriptive fuzzy inference procedure in the form of if-then statements. In general, a fuzzy if-then rule has two constitutes; first the if part and the second the then part; which are called premise and consequent, respectively. The general form of a fuzzy if-then

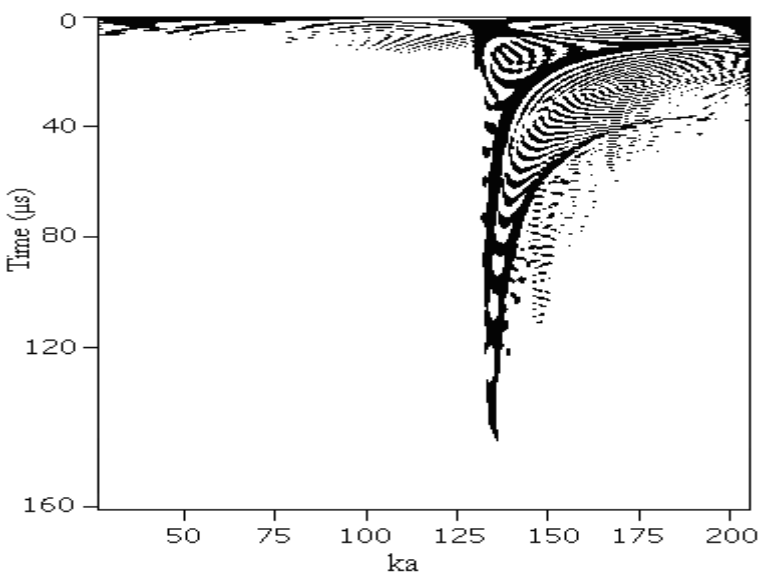

Fig.7. $\quad S P W V$ of backscattered signal for the second aluminum cylindrical shell of radii radio $b / a=0.95$ (Anti-symmetric circumferential wave $A 1$, $130<k a<200$ )

rule is as follows; Rule: if $Z$ is $A$ then $f$ is $B$.

2) A database, which defines the membership functions of the fuzzy sets used in the fuzzy rules.

3) A decision-making unit, which performs the inference operations on the rules.

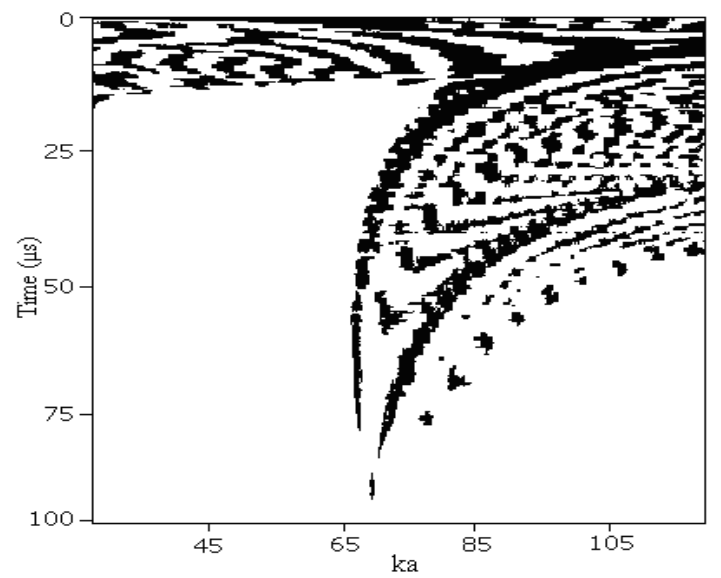

Fig.8. $\quad S P W V$ of backscattered signal for the third aluminum cylindrical shell of radii radio $b / a=0.9$ (Anti-symmetric circumferential wave $A l$, $65<k a<120$ )

4) A fuzzification inference, which transforms the crisp inputs into degree of match with linguistic values.

5) A defuzzification inference, which transforms the fuzzy results of the inference into a crisp output.

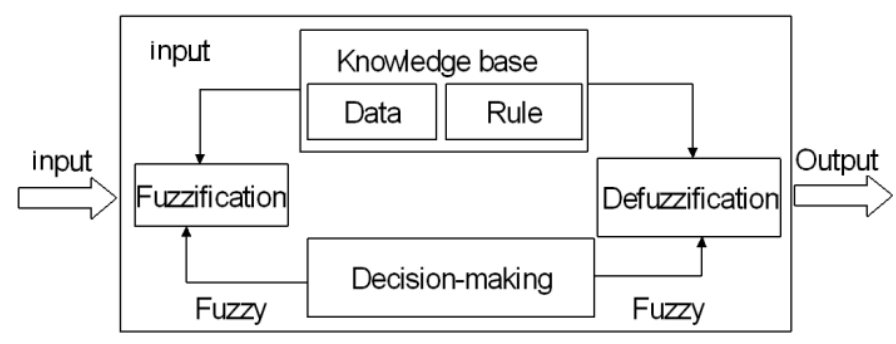

Fig.9. Bloc diagram for a fuzzy Inference System

Several types of FIS have been proposed in the literature[29], which, vary due to differences between the specification of the consequent part and the defuzzification schemes. This paper incorporates one of these types, the socalled Takagi and Sugeno FIS [30], to propose a systematic scheme for the development of fuzzy rules using the input/output data sets.

A typical fuzzy rule in a sugeno fuzzy model has the format:

If $x$ is $A$ and $y$ is $B$ then $z=f(x, y)$

where $A$ and $B$ are fuzzy sets in the antecedent; $z=f(x, y)$ is a crisp function in the consequent. Usually $f(x, y)$ is a polynomial in the input variable $x$ and $y$, but it can be any other functions that can appropriately describe the output of the system within the fuzzy region specified by the antecedent of the rule. When $f(x, y)$ is a first order polynomial, we have the first-order sugeno fuzzy model. When $f$ is a constant, we then have the zero-order Sugeno fuzzy model. Consider firstorder Sugeno fuzzy inference systems which contain two rules:

Rule 1: if $x$ is $A_{1}$ and $y$ is $B_{1}$ then $f_{1}=p_{1} x+q_{1} y+r_{1}$.

Rule 2: if $x$ is $A_{2}$ and $y$ is $B_{2}$ then $f_{2}=p_{2} x+q_{2} y+r_{2}$.

Weighted averages are used in order to avoid complexity in deffuzification processes. Figure 10 illustrates graphically the fuzzy reasoning mechanism to derive an output $f$ from a given input vector $(x, y)$. The firing strengths $\omega_{1}$ and $\omega_{2}$ are usually obtained as the product of the membership grades in the premise part, and the output $f$ is the weighted average of each rule's output. To facility the learning of the sugeno fuzzy model, into the framework of adaptative networks we can compute gradient vectors systemically. The resultant network architecture is called Adaptative Neuro Fuzzy Inference system (ANFIS).

\section{B. Adaptive neuro-fuzzy inference system architecture}

The Adaptive Network-based Fuzzy Inference System (ANFIS) is developed by Jang in 1993 [26]. This model use neuro-adaptive learning techniques, which are similar to those of neural networks. Given an input/output data set, the ANFIS can construct a Fuzzy Inference System whose membership function parameters were adjusted using a hybrid algorithm learning that is a combination of Last Square estimate and the gradient descent back-propagation algorithm or other similar optimisation technique. This allows Fuzzy system to learn from the data they are modelled. 
For simplicity, we assume the fuzzy inference system with two input, $x$ and $y$ with one response $f$. From the first-order Sugeno fuzzy model, a typical rule set with two fuzzy if-then rules can expressed as below. The corresponding equivalent ANFIS architecture is as shown in figure 11. The system architecture consists of five layers, namely; fuzzy layer, product layer, normalized layer, fuzzy layer and total output layer. The following section in depth the relationship between the input and output of each layer in ANFIS.

Layer 0: It consists of plain input variable set.

Layer 1: It is the fuzzy layer. Each node in this layer generates a membership grade of a linguistic label. For instance, the node function of the $\mathrm{i}^{\text {th }}$ node may be generalized bell membership function:

$$
\mu_{A_{i}}=\frac{1}{1+\left[\frac{x-c_{i}}{a_{i}}\right]^{b_{i}}}
$$

where $x$ is the input to node $i ; A_{i}$ is the linguistic label (small, large, etc.) associated with this node; and $\left\{a_{i}, b_{i}, c_{i}\right\}$ is the parameter set that changes the shapes of the membership function. Parameters in this layer are referred to as the premise parameters.

Layer 2: The function is T-norm operator that performs the firing strength of the rule, e.g., fuzzy conjective AND and OR. The simplest implementation just calculates the product of all incoming signals.

$$
\omega_{i}=\mu A_{i}(x) \mu B_{i}(\mathrm{y}), \mathrm{i}=1,2
$$

Layer 3: Every node in this layer is fixed and determines a normalized firing strength. It calculates the ratio of the ratio of the $\mathrm{j}^{\text {th }}$ rule's firing strength to the sum of all rules firing strength.

$$
\varpi_{i}=\frac{\omega_{i}}{\omega_{1}+\omega_{2}}, i=1,2
$$

Layer 4: The nodes in this layer are adaptive are connected with the input nodes and the preceding node of layer 3. The result is the weighted output of the rule $j$.

$$
\varpi_{i} f_{i}=\omega_{i}\left(p_{i} x+q_{i} y+r_{i}\right)
$$

where $\varpi_{i}$ is the output of layer 3 and $\left\{p_{i}, q_{i}, r_{i}\right\}$ is the parameter set. Parameters in this layer are referred to as the consequent parameters.

Layer 5: This layer consists of one single node which computes the overall output as the summation of all incoming signals.

$$
\text { Overall Output } \sum_{i} \varpi_{i} f_{i}=\frac{\sum_{i} \omega_{i} f_{i}}{\sum_{i} \omega_{i}}
$$

The constructed adaptive network in figure 11 is functionally equivalent to a fuzzy inference system in figure
10. The basic learning rule of ANFIS is a combination of last squar error and the back-propagation gradient descent, which calculates error signals (the derivative of the squared error with respect to each node's output) recursively from the output layer backward to the input nodes. This learning rule is exactly the same as the back-propagation learning rule used in the common feed-forward neural networks.

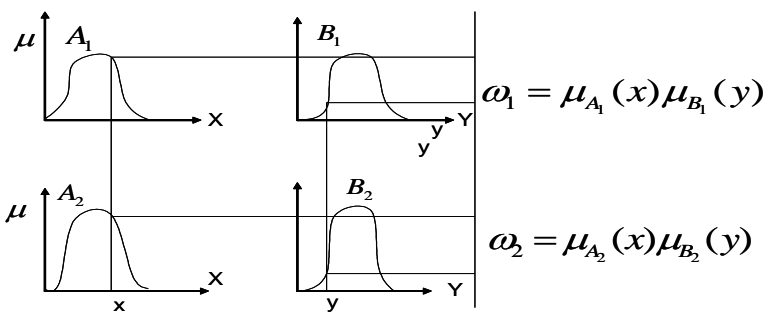

Fig.10. First-order Sugeno fuzzy model

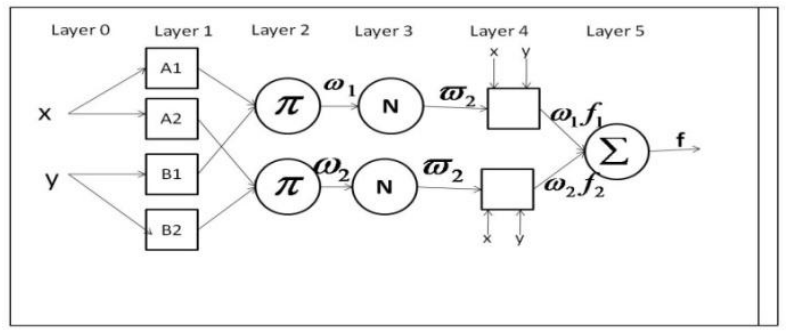

Fig.11. ANFIS architecture

\section{COLLECTION Of DATA}

The conception of the fuzzy logic model requires the determination of the relevant entries that have a significant influence on the required model. In this work, a data base is collected to involve and test the performance of the model starting from the results obtained by the time-frequency of Wigner-Ville method then supplemented by data resulting from the proper modes theory of the circumferential waves. The density of material, the radius ratio, the index of the antisymmetric and symmetric circumferential waves, and longitudinal and transverse velocities, of the material constituting the cylindrical shell, are retained like relevant entries of the model because these parameters characterize the cylindrical shell and the types of circumferential waves propagating around this one. The cut-off frequency $(k a)_{c}$, of the anti-symmetric and symmetric circumferential waves $\left(S_{\mathrm{i}}\right.$ and $\left.A_{i}, i=1,2\right)$ for a aluminum cylindrical shell with different radius ratios $b / a$, constitutes the output of fuzzy system. The collected data for the training and validation phases of the fuzzy logic system model are represented in tables I and II. For example, for aluminum cylindrical shell, the density is $2700 \mathrm{~kg} / \mathrm{m}^{3}$, the transverse velocity is $3100 \mathrm{~m} / \mathrm{s}$ and the longitudinal velocity is $6380 \mathrm{~m} / \mathrm{s}$. For the anti-symmetric circumferential wave $A 1$ the cut-off frequency is 132.43 for a radius ratio $b / a$ equal to 0.95 .

\section{RESUlTS AND DISCUSSION}

The performance of ANFIS models for training and testing data sets were evaluated according to statistical criteria such as, coefficient of correlation $R, M A E, M R E, S E$, and root mean square error (RMSE). The selection of different models is done 
comparing the errors of the ANFIS configuration, calculating the $M A E$, the $M R E$ and the $S E$ of the cut-off frequency. The coefficient of correlation $R$ and the determination $R^{2}$ of the linear regression are used like performance measures of the model between the predicated and the desired output. The different error measures and the coefficient of correlation are given by the following relations:

$$
\begin{aligned}
& \text { MAE }=\frac{1}{n} \sum_{i=1}^{n}\left|D_{i}-P_{i}\right| \\
& M R E=\frac{1}{n} \sum_{i=1}^{n} \frac{\left|D_{i}-P_{i}\right|}{D_{i}} \\
& R=1-\frac{\sum_{i=1}^{n}\left(D_{i}-P_{i}\right)^{2}}{\sum_{i=1}^{n}\left(D_{i}-P_{m}\right)^{2}} \\
& S E=\frac{\sqrt{\sum_{i=1}^{n}\left(D_{i}-P_{i}\right)^{2}}}{n-1}
\end{aligned}
$$

where $n$ is the number of data, $P_{i}$ and $D_{i}$ is the predicted and desired of cut-off frequency respectively and $P_{m}$ is the mean of predicted values.

The coefficient of correlation is a commonly used statistic and provides information on the strength of linear relationship between the observed and the computed values. The training and testing performances of ANFIS models are given in figures 12 to 15 .

The analysis is repeated several times. Indeed, the error values are measured for each ANFIS architecture based on the number of rules and the type of the membership function used. In this work we tried to play on the number of rules and the number of epochs we have observed that the error values of our models decrease more than the number of rules, and the number of epochs is increases. The results of the measured errors are presented in figures 12 to 15 for each circumferential wave $(A 1, S 1, S 2$ and $A 2)$. Tables V to VI show that the results obtained by the fuzzy system method are in good agreement with those determined from the results calculated using the proper modes theory of resonances, and they are better to those determined manually from the timefrequency of Wigner-Ville images (Table V).

TABLE V. RESULTS OF THE CUT-OFF FREQUENCIES OF MODE Al OBTAINED BY THE ANFIS MODEL, THE PROPER MODES THEORY AND BY THE TIME-FREQUENCY OF WIGNER-VILLE IMAGES

\begin{tabular}{|c|c|c|c|}
\cline { 2 - 4 } \multicolumn{1}{c|}{} & \multicolumn{3}{c|}{ Cut-off frequencies $(\boldsymbol{k a})_{\boldsymbol{c}}$} \\
\hline $\begin{array}{c}\text { Cylindrical } \\
\text { shell }\end{array}$ & $\begin{array}{c}\text { Computed } \\
\text { using } \boldsymbol{P M T}\end{array}$ & $\begin{array}{c}\text { Determined } \\
\text { using } \boldsymbol{A N F I S}\end{array}$ & $\begin{array}{c}\text { Determined } \\
\text { using } \boldsymbol{S P W V} \boldsymbol{V}\end{array}$ \\
\hline $\begin{array}{c}\boldsymbol{b} / \boldsymbol{a}=\mathbf{0 . 9} \text { (figure } \\
\text { 8) }\end{array}$ & 66.21 & 66.16 & $66.0 \pm 0.3$ \\
\hline $\begin{array}{c}\boldsymbol{b} / \boldsymbol{a}=\mathbf{0 . 9 5} \text { (figure } \\
\text { 7) }\end{array}$ & 132.43 & 132.59 & $132.0 \pm 0.3$ \\
\hline $\begin{array}{c}\boldsymbol{b} / \boldsymbol{a}=\mathbf{0 . 9 7} \text { (figure } \\
\text { 6) }\end{array}$ & 220.72 & 221.32 & $221.0 \pm 0.2$ \\
\hline
\end{tabular}

\begin{tabular}{|c|c|c|c|c|c|c|}
\hline & \multicolumn{6}{|c|}{ (ka)c computed and determined } \\
\hline & ANFIS & PMT & ANFIS & PMT & ANFIS & PMT \\
\hline & \multicolumn{2}{|c|}{$\mathrm{b} / \mathrm{a}=0.9$} & \multicolumn{2}{|c|}{$\mathrm{b} / \mathrm{a}=0.95$} & \multicolumn{2}{|c|}{$\mathrm{b} / \mathrm{a}=0.97$} \\
\hline $\begin{array}{c}\text { Mode } \\
\text { A1 }\end{array}$ & 66.16 & 66.21 & 132.59 & 132.43 & 221.32 & 220.72 \\
\hline $\begin{array}{c}\text { Mode } \\
\text { S1 }\end{array}$ & 132.32 & 132.43 & 265.17 & 264.87 & 446.64 & 441.45 \\
\hline $\begin{array}{c}\text { Mode } \\
\text { S2 }\end{array}$ & 136.16 & 136.28 & 272.87 & 272.56 & 459.60 & 454.26 \\
\hline $\begin{array}{c}\text { Mode } \\
\text { A2 }\end{array}$ & 198.51 & 198.65 & 398.49 & 397.30 & 668.95 & 662.17 \\
\hline
\end{tabular}

TABLE VI. RESULTS OF THE CUT-OFF FREQUENCIES OF DIFFERENT MODES OBTAINED BY THE ANFIS MODEL AND THE PROPER MODES THEORY PMT FOR THE CYLINDRICAL SHELL

The results of the different error measures and the coefficient of correlation (MRE, MAE, SE and R) are given in the table VII. And also are illustrated on the Figs. 12a to 15a. So, it is interest to use the approach of the Fuzzy Logic. The best configuration is found for a network with 13 rules. The predicted values are traced according to the desired values in the figures 12 to 15 ((a), (b), and (c)). The results show the good agreement between the predicted and the desired values of the cut-off frequency. The coefficient of determination $R^{2}$ for this optimal configuration is 1 (Figs. 12(a) to 15 (a)). Figs. 12 to 15 ( $a$ and $b$ ) show that the cut-off frequency increases rapidly when the radius ratio $b / a$ of the cylindrical shell tends to one. The evolution of the mean quadratic errors (RMSE) of training during the training phase is illustrated on Fig. 16.

TABLE VII. RESULTS OF THE DIFFERENT ERROR MEASURES AND THE COEFFICIENT OF CORRELATION (MRE, MAE, SE AND R) WITH 13 RULES

\begin{tabular}{|c|c|c|c|c|}
\hline $\begin{array}{c}\text { Error } \\
\text { measures }\end{array}$ & Mode A1 & Mode S1 & Mode S2 & Mode A2 \\
\hline MAE & $0.03 \mathrm{ka}$ & $0.08 \mathrm{ka}$ & $0.07 \mathrm{ka}$ & $0.07 \mathrm{ka}$ \\
\hline MRE & $0.810^{-3} \mathrm{ka}$ & $0.810^{-3} \mathrm{ka}$ & $0.810^{-3} \mathrm{ka}$ & $0.510^{-3} \mathrm{ka}$ \\
\hline $\mathrm{SE}$ & $910^{-3} \mathrm{ka}$ & $10^{-2} \mathrm{ka}$ & $210^{-2} \mathrm{ka}$ & $10^{-2} \mathrm{ka}$ \\
\hline $\mathrm{R}=\mathrm{R}^{2}$ & 1 & 1 & 1 & 1 \\
\hline
\end{tabular}

\section{CONCLUSION}

The main aim of this work was to train an ANFIS model to predict cut-off frequency with the minimum of input data. Results show that the trained model can be used as an alternative way in the modelling behaviour system. This fuzzy logic model taking into account some characteristics of the tube is developed in order to predict the cut-off frequency for various types of circumferential waves $A_{1}, S_{1}, S_{2}, A_{2}$. In this article, this model is applied to aluminum tubes. This model can be used to predict the evolution of the group and phase velocities according to the frequency. It also can constitute a help for the estimate of various parameters of a tube starting from the characteristics of which it is disposed.

The use of the fuzzy logic does not present any approximation as in the case of the natural modes method which assimilates the tubes to the plates with the same thickness and that is not sullied with errors as in the case of the time-frequency representations of Wigner-Ville that determines the cut-off dimensionless frequency manually 
starting from the time-frequency image. This article can be used as a new tool for characterization of an elastic tube. This method allows one to determine automatically and with good
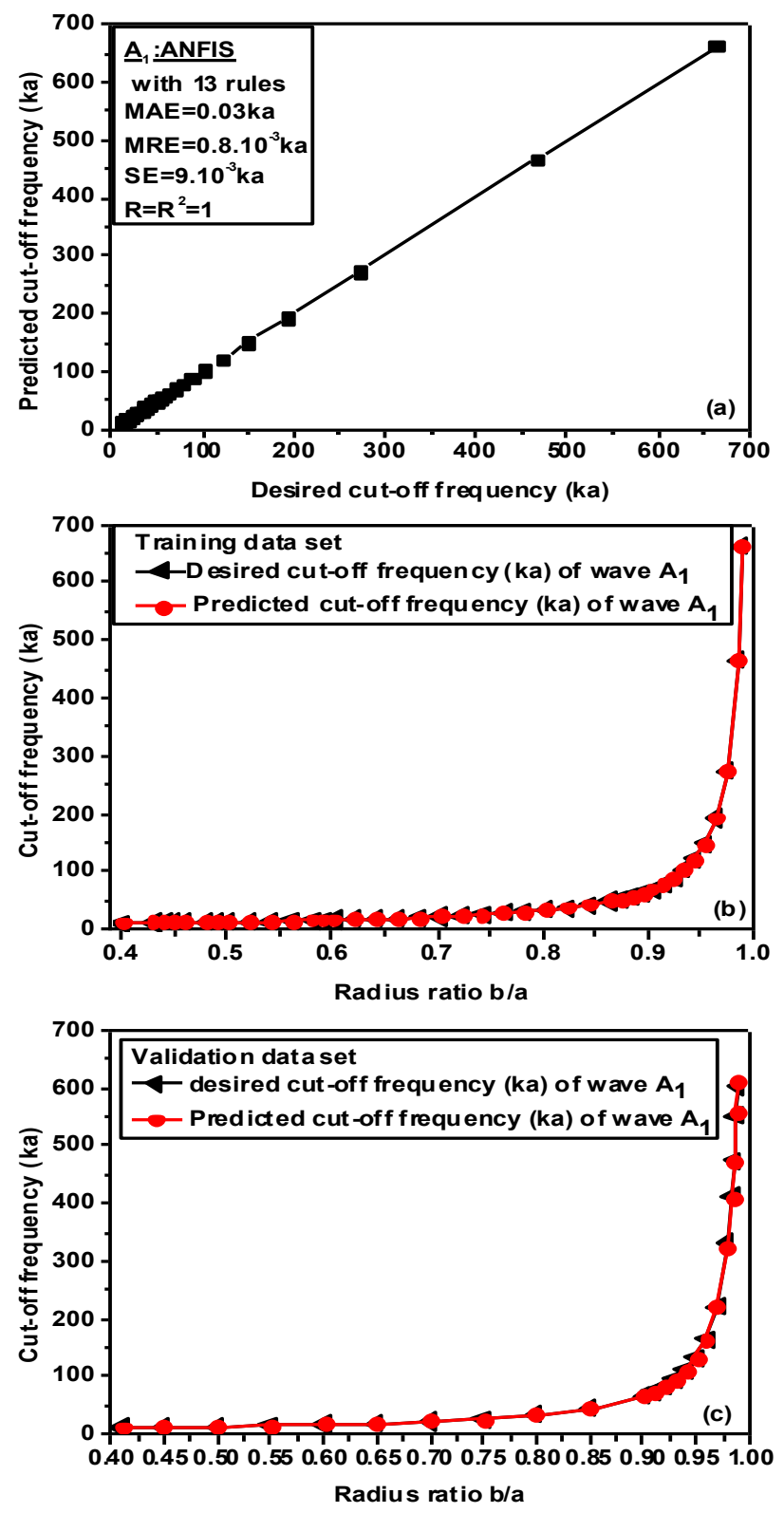

Fig.12. (a) Correlation of desired versus ANFIS values of cut-off frequency of anti-symmetric wave $A l$ with validation data set, (b) Cut-off frequency as a function of radius ratio of aluminum cylindrical shell on training data set and (c) Cut-off frequency as a function of radius ratio of an aluminum cylindrical shell on validation data set precision the reduced cut-off frequency of an antisymmetric wave propagating around the tube. The $\mathrm{R}^{2}$ value in fig is about 1, which can be considered as very satisfactory.
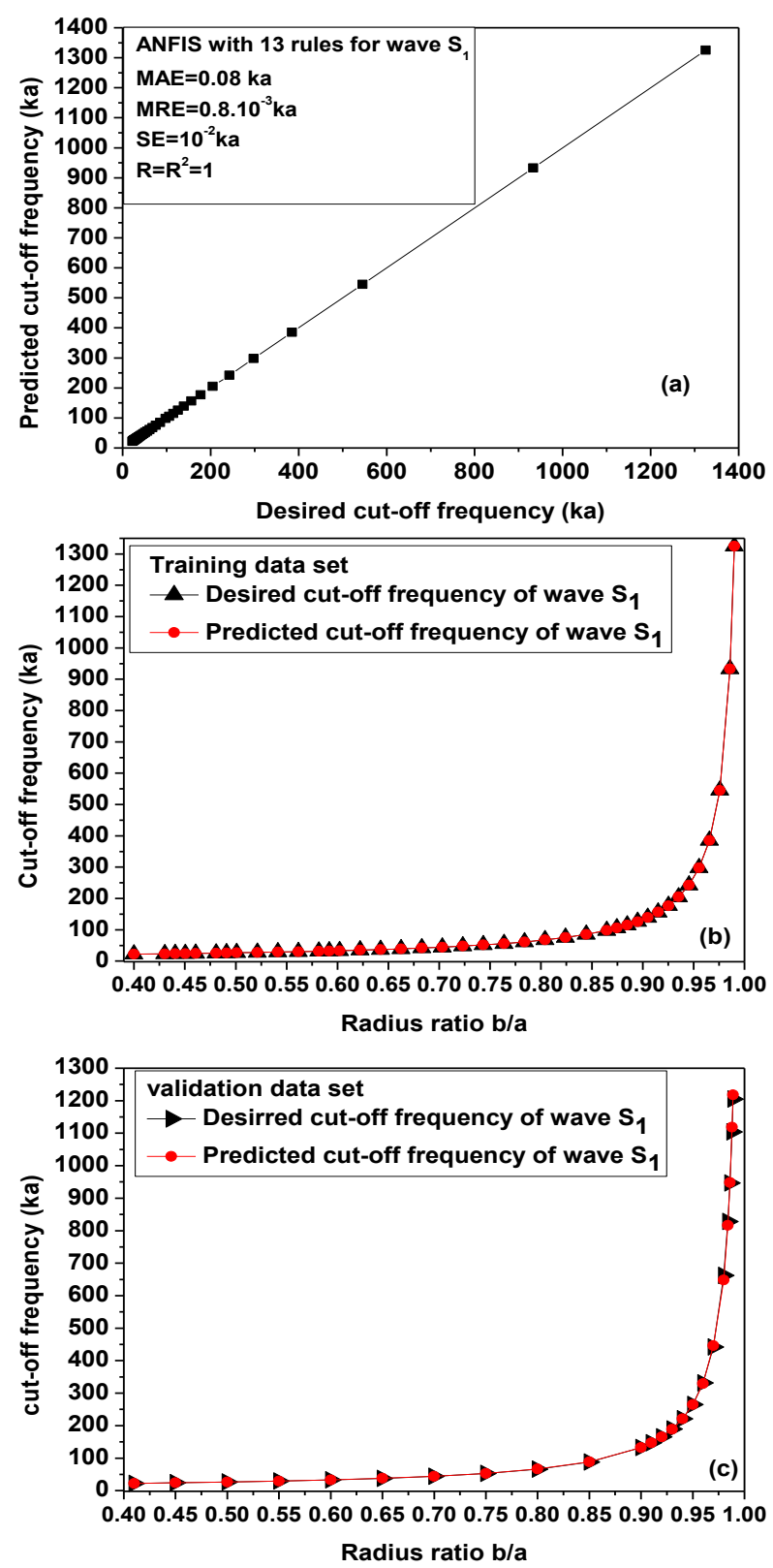

Fig.13. (a) Correlation of desired versus ANFIS values of cut-off frequency of Symmetric wave $S 1$ with validation data set, (b) Cut-off frequency as a function of radius ratio of aluminum cylindrical shell on training data set and (c) Cut-off frequency as a function of radius ratio of an aliminum cylindrical shell on validation data set 

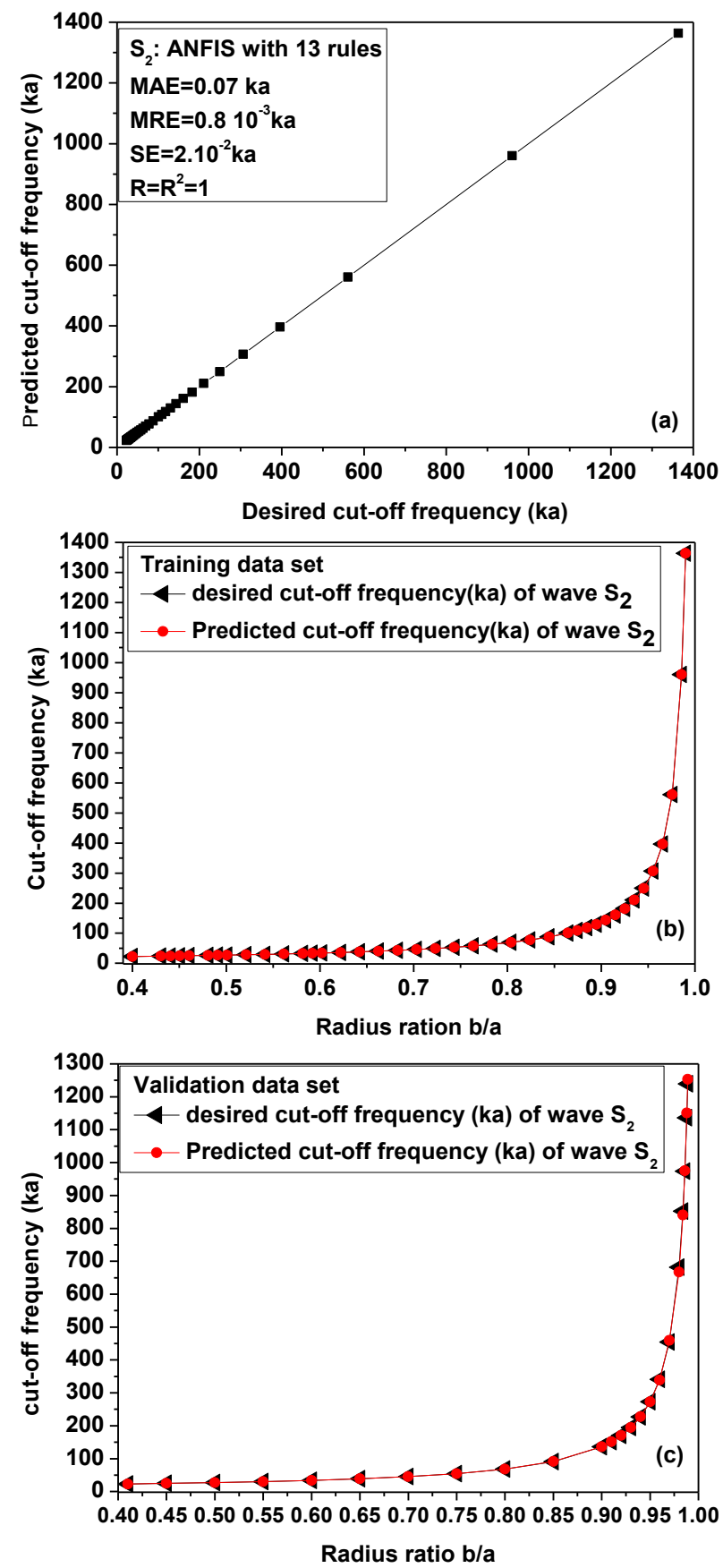

Fig.14. (a) Correlation of desired versus ANFIS values of cut-off frequency of Symmetric wave $S 2$ with validation data set, (b) Cut-off frequency as a function of radius ratio of aluminum cylindrical shell on training data set and (c) Cut-off frequency as a function of radius ratio of an aliminum cylindrical shell on validation data set
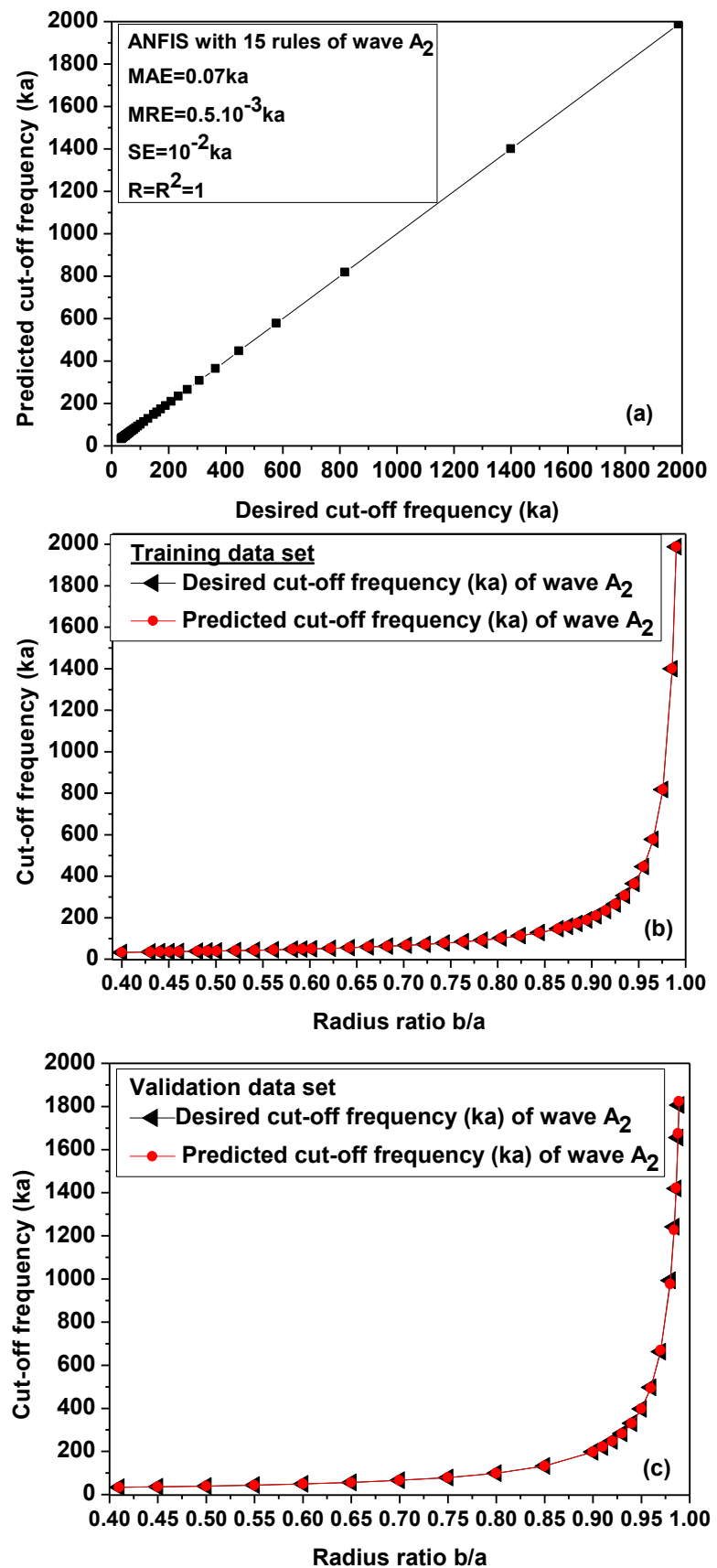

Fig. 15. (a) Correlation of desired versus ANFIS values of cut-off frequency of Anti-symmetric wave A2 with validation data set, (b) Cut-off frequency as a function of radius ratio of aluminum cylindrical shell on training data set and (c) Cut-off frequency as a function of radius ratio of an aluminum cylindrical shell on validation data set 

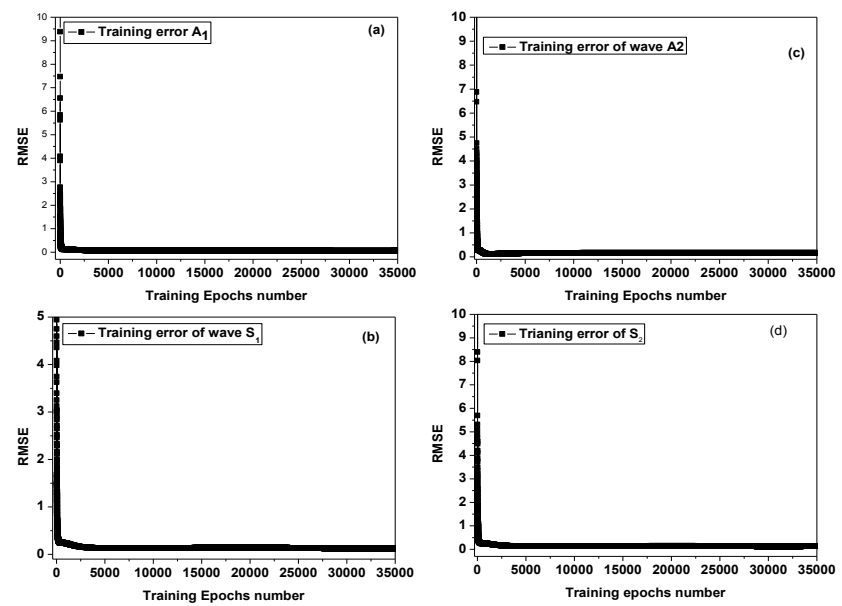

Fig.15. Visualization of errors of training and testing as a function of the number of iteration for an ANFIS to four entries and tree rules. Antisymmetric and Symmetric waves (a) A1, (b) S1, (c) S2, (d) A2

\section{REFERENCES}

[1] R. Latif, E. Aassif, A. Moudden, D. Decultot, B. Faiz, and G. Maze, "Determination of the cut-off frequency of an acoustic circumferential wave using a time-frequency analysis," J. NDT\&E Int., vol. 33, pp. 373$376,2000$.

[2] R. Latif, E. Aassif, M. Laaboubi, G. Maze, "Détermination de l'épaisseur d'un tube élastique à partir de l'analyse temps-fréquence de Wigner-Ville", Acta Acustica united with Acustica, Vol. 95, pp. 253257, (2009)

[3] M. Talmant, J. L. Izbicki, G. Maze, G. Quentin J. Ripoche. "External wave resonance on thin cylindrical shells". J. Acoustique, 4, pp. 509523(1991).

[4] L. Haumesser, D. Décultot, F. Léon, and G. Maze, "Experimental identification of finite cylindrical shell vibration modes", Journal of the Acoustical Society of America, Vol. 111, 5, pp. 2034-2039, (2002)

[5] J. D. N. Cheeke, X. Li, and Z. Wang, "Observation of flexural Lamb waves (A0 mode) on water-filled cylindrical shells", Journal of the Acoustical Society of America, Vol. 104, 6, pp. 3678-3680, (1998).

[6] P. L. Marston and N. H. Sun, "Backscattering near the coincidence frequency of a thin cylindrical shell: Surface wave properties from elastic theory and an approximate ray synthesis," J. Acoust. Soc. Amer., vol. 97, pp. 777-783, 1995.

[7] G. Maze, "Acoustic scattering from submerged cylinders. MIIR Im/Re: Experimental and theoretical study," J. Acoust. Soc. Amer., vol. 89, pp. 2559-2566, 1991.

[8] R. Latif, E. Aassif, G. Maze, A. Moudden, B. Faiz, "Determination of the group and phase velocities from time-frequency representation of Wigner-Ville", Journal of Non Destructive Testing \& Evaluation International, Vol.32, 7, pp. 415-422, (1999)

[9] G. Kaduchak, C. S. Kwiatkowshi, and P. L. Marston, "Measurement and interpretation of the impulse response for backscattering by a thin spherical shell using a broad bandwidth source that is nearly acoustically transparent," J. Acoust. Soc. Amer.,vol. 97, pp. 2699-2708, 1997.

[10] P. Flandrin, Temps-frequence. Paris: Hermès, 1993.

[11] E. Aassif, R. Latif, D. Decultot, G. Maze, B. Faiz, and A. Moudden, "Time-frequency analysis of the complex pressure scattered by immersed tubes," in 3rd Int. Conf., Acoust. Vibratory Surveillance Methods Diagnostic Techniques, Centre Technique des Industries Mécaniques, CETIM-Senlis, France, Oct. 13-15, 1998, pp. 471-480.
[12] R. Latif, E. H. Aassif, A. Moudden, and G. Maze, "Analyse des caractéristiques acoustiques d'une plaque élastique immergée dans l'eau à partir de l'image temps-fréquence," Acta Acustical Acustica, vol. 92, pp. 549-555, 2006.

[13] S. F. Morse and P. L. Marston, "Backscattering of transients by tilted truncated cylindrical shells: Time-frequency identification of ray contributions from measurements," J. Acoust. Soc. Amer., vol. 111, pp. 1289-1294, 2002.

[14] R. LATIF, E. AASSIF, G. MAZE, D. DECULTOT, A. MOUDDEN, B. FAIZ, "Analysis of the circumferential acoustic waves backscattered by a tube using the time-frequency representation of Wigner-Ville", Journal of Measurement Science and Technology, Vol. 11, 1, pp. 83-88, (2000).

[15] R. LATIF, E. AASSIF, A. MOUDDEN, B. FAIZ, "Caractérisation ultrasonore d'un matériau élastique à partir de l'analyse de l'image temps-fréquence de Wigner-Ville ", Acta Acustica united with Acustica, Vol. 89, pp. 253-257, (2003).

[16] D. H. Hughes and P. L. Marston, "Local temporal variance of Wigner's distribution function as a spectroscopic observable: Lamb wave resonances of a spherical shell," J. Acoust. Soc. Amer., vol. 94, pp. 499505, 1993.

[17] R. Latif, E. Aassif, M. Laaboubi, G. Maze, "Determination of group velocity using the time-varying Autoregressive TVAR", Conference international IEEE ISSPA, Sharjah UAE (2007).

[18] R. Latif, M. Laaboubi, E. Aassif, A. Moudden, G. Maze, "Determination of the cut-off frequency of the anti-symmetric circumferential waves using the time-varying autoregressive TVAR“, 3rd International Symposium on Communications, Control and Signal Processig IEEE ISCCSP'08, pp. 357-361, St Julians Malta, 12-14 March (2008)

[19] T. Onsay and A. G. Haddow, "Wavelet transform analysis of transient wave propagation in a dispersive medium", J. Acoust. Soc. Am. Vol. 95, pp. 1441-1449, (1994)

[20] K. Kishimoto, H. Inoue, M. Hamada and T. Shibuya, "Time-frequency analysis of a dispersive waves by means of wavelet transform", ASME J. Appl. Mech. Vol. 62, pp. 841-846, (1995)

[21] A. Dariouchy, E. Aassif, G. Maze, R.Latif, D. Decultot, M. Laaboubi, "Prediction of the Acoustic Pressure Backscattered by a Steel Tube Using Neural Networks Approach", International Symposium on Computational Intelligence and Intelligent Informatics ISCIII'07, Agadir Morocco, 28-30 March (2007)

[22] A. Dariouchy, E. Aassif, G. Maze, D. Décultot, A. Moudden, "Prediction of the acoustic form function by neural network techniques for immersed tubes", J. Acoust. Soc. Am. Vol. 124, 2, pp. 1018-1025 (2008)

[23] G. Maze, J. Ripoche, A. Derem, J. L. Rousselot, "Diffusion d'une onde ultrasonore par des tubes remplis d'air immergés dans l'eau", Acustica, vol. 55, pp. 69-85, (1984).

[24] Younho Cho, "Estimation of ultrasonic guided wave mode conversion in a plate with thickness variation", Ultrasonics, Ferroelectrics and Frequency Control, IEEE Transactions, Vol. 47, 3, pp. 591-603, (2000).

[25] Atkinson D., Hayward G., "Embedded acoustic fibre wave guides for Lamb wave condition monitoring", Ultrasonics Symposium, IEEE, Vol. 1, pp. 699-702, (1999).

[26] Jang J-SR. ANFIS: Adaptive-network-based fuzzy inference systems. IEEE Trans Syst Man Cybern 1993; 23(3):665-85.

[27] S. Horikawa, T. Furuhashi, and Y Uchikawa. IEEE Trans. Neural Networks 3:801-806, 1992.

[28] H. Ishibuchi, R. Fujioka, and H. Tanaka. IEEE Trans. Fuzzy Systems 1:85-97, 1993.

[29] Lee CC. Fuzzy logic in control system: Fuzzy logic controller-part I and part II. IEEE Trans Syst Man Cybern 1990; 20(2):404-35.

[30] Sugeno M. Industrial applications of fuzzy control. New York: Elsevier Ltd.; 1985. 